\title{
Determining Surface Stimulation Parameters With Computational Cardiac Electrophysiology to Defibrillate Human Ventricles
}

\author{
Angel Moreno ${ }^{1,2}$, Richard D Walton ${ }^{1,3}$, Claudia Hawks ${ }^{4}$, Olivier Bernus ${ }^{1,3}$, Edward J Vigmond ${ }^{1,2}$, \\ Jason D Bayer ${ }^{1,2}$ \\ ${ }^{1}$ Electrophysiology and Heart Modeling Institute (IHU LIRYC), Bordeaux University Foundation, \\ Bordeaux, France \\ ${ }^{2}$ Institute of Mathematics of Bordeaux (UMR 5251), University of Bordeaux, Bordeaux, France \\ ${ }^{3}$ Cardio-Thoracic Research Center of Bordeaux (INSERM U1045), University of Bordeaux, \\ Bordeaux, France \\ ${ }^{4}$ Department of Applied Physics and Mathematics, University of Navarra, Pamplona, Spain
}

\begin{abstract}
Aim: To determine the minimal surface stimulus strength necessary to defibrillate human ventricles.

Methods: We determined this in a $10 \mathrm{~cm} x 10 \mathrm{~cm} x 1 \mathrm{~cm}$ finite element model of left ventricular (LV) tissue with human electrophysiology. It was paced until steady-state at $1.3 \mathrm{~Hz}$ with S1 stimuli (LV apex) at $2 x$ threshold. A single S2 stimulus (apical posterior corner) was delivered at $2 x$ threshold $300 \mathrm{~ms}$ after the last $\mathrm{S1}$ to initiate reentry at the model's center. To terminate reentry, an S3 stimulus on the entire epicardial, endocardial, or both surfaces was applied 2 seconds after S2. Starting at the capture threshold, $S 3$ strength was doubled until reentry terminated.

Results: Minimum stimulus strength to terminate reentry with endocardial and epicardial S3 was $>512$-fold capture threshold, but only 16-fold for S3 applied to both surfaces simultaneously. Extending the S3 depth to the midmyocardium lowered the minimum stimulus strength for defibrillation to 8-fold capture threshold on the endocardium and 16-fold on the epicardium. This difference corresponds to a longer wavelength for reentry in the endocardium versus epicardium $(6.6 \mathrm{~cm}$ vs $5.3 \mathrm{~cm})$.

Conclusion: Stimulating both the endocardial and epicardial surfaces at $\geq 16$ times capture threshold is optimal to defibrillate human ventricles, and is decreased further for stimuli applied deeper in the tissue.
\end{abstract}

\section{Introduction}

\subsection{Background}

Ventricular fibrillation (VF) is the most deadly cardiac arrhythmia. The disorganized electrical activity in the ven- tricles underlying VF leads to cardiac arrest and sudden cardiac death. Consequently, sudden cardiac death from VF is a leading cause of death throughout the world [1].

The most effective life-saving therapy for defibrillating the ventricles, i.e. terminating $\mathrm{VF}$ and subsequently resetting the heart beat back to a normal sinus rhythm, is a farfield direct-current electrical shock. Unfortunately, such shocks are very strong (several Joules) and cause extreme pain, phobic anxiety disorders, and irreversible cardiac tissue damage that increases mortality [2].

To circumvent these limitations for strong electric shocks delivered to the chest and body, optogenetics [3] and conformal electronics [4] have shown promise for terminating VF with less pain and damage via direct cardiac surface stimulation. However, the application of these technologies to defibrillate large mammalian ventricles is not well established. Specifically, it is unknown at what stimulus strength ventricular surface stimulation robustly terminates VF in the human ventricles, and on which ventricular surfaces (endocardium, epicardium, or both) the stimulation needs to be administered.

\subsection{Aims}

This study aims to determine: i.) the minimum stimulus strength required to terminate $\mathrm{VF}$ in the human ventricles with unipolar surface stimulation; ii.) how the minimum stimulus strength varies with respect to the surfaces stimulated; and iii.) how stimulus depth in the tissue alters the minimum stimulus strength. Since performing this study in human patients is dangerous and unethical, and in large animals expensive and time-consuming, we used computational cardiac electrophysiology [5], which is an ethical and cost-effective mean to rapidly study cardiac electrophysiology from the cell to organ level. 


\section{Methods}

\subsection{LV wedge model}

Ventricular defibrillation was simulated and studied in a large $10 \mathrm{~cm} \times 10 \mathrm{~cm} \times 1 \mathrm{~cm}$ model of left ventricular (LV) tissue. This volume was discretized at $100 \mu \mathrm{m}$ with 800,000 hexahedra finite elements and 848,421 nodes. Fiber orientation was implemented into each element of the model using a rule-based approach [6] to match the LV fiber directions and normal conduction velocities in Bayer et al [7]. At the cellular level, membrane kinetics were described with a modified ten Tusscher-Panfilov human ventricular myocyte model [8], which includes transmural heterogeneity found in normal human hearts using the parameter settings in Bayer et al [7].

All simulations were performed with the Cardiac Arrhythmia Research Package (https://carp.medunigraz.at/) and were monodomain using a time step of $20 \mu$ s on four compute nodes each with two Hexa-Core Intel Xeon X5675 @ 3.06GHz CPUs and 48 GB of memory.

\subsection{VF initiation protocol}

The entire apical surface (planar wave propagation) of the LV tissue model was paced until steady-state at 1.3 $\mathrm{Hz}$ with 2 ms long $\mathrm{S} 1$ transmembrane current stimuli at twice capture threshold. Reentry at the center of the LV tissue model was generated with a single $\mathrm{S} 2$ transmembrane current crossfield stimulus delivered $300 \mathrm{~ms}$ following the $\mathrm{S} 1$. The S2 was $2 \mathrm{~ms}$ long at twice capture threshold, consisting of all model nodes in the apical posterior quadrant of the LV tissue model (Figure 1.A). A pseudo-ECG was computed during reentry according to Gima et al [9] between two points $3 \mathrm{~cm}$ away from the centers of the epicardial and endocardial surfaces. The dominant frequency of the pseudo-ECG was computed according to Berenfeld et al [10].

\subsection{VF defibrillation protocol}

To terminate reentry, a single $2 \mathrm{~ms}$ long S3 stimulus was applied two seconds after the S2. The S3 was applied uniformly over the epicardial, endocardial, or both surfaces. For each of the three stimulus configurations, the S3 stimulus strength was initially set to the capture threshold for each surface determined at the end of S1 in the VF initiation protocol, and then doubled until reentry in the LV tissue model terminated within one second after administering S3. The doubling was stopped after 512-fold increases due to the instability of the sodium channel in the ventricular myocyte model at these stimulus strengths. To determine how stimulus strength depends on transmural depth, the defibrillation protocol was repeated by expanding the surface simulation to $50 \%$ the transmural depth from the given surface. Thus, when stimulating both surfaces it will be $100 \%$ of the tissue volume being stimulated.

\section{Results}

\subsection{VF initiation}

The reentry generated in the LV wedge model with the VF initiation protocol (Figure 1.B) had a dominant frequency of $5.95 \mathrm{~Hz}$ with a mean cycle length of $168 \mathrm{~ms}$, which is within the range seen clinically for VF onset [11].
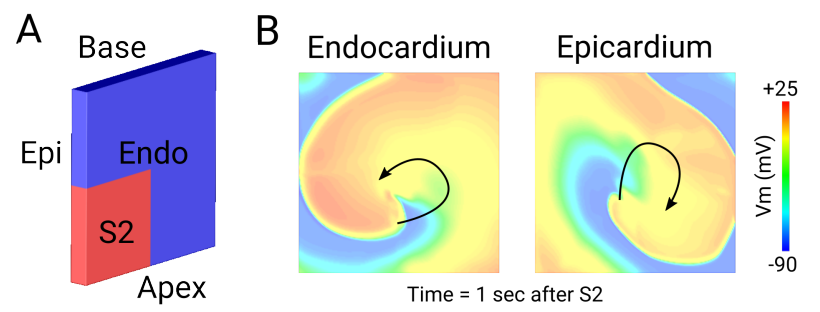

Figure 1. VF initiation in LV wedge model. A. S2 stimulus for initiating VF. $B$. Endocardial and epicardial views of reentry at the moment in time the defibrillation protocol is administered.

\subsection{Defibrillation}

The S3 stimulus threshold for capture during diastole on both the endocardial and epicardial surfaces was the same at $14 \mu \mathrm{A} / \mathrm{cm}^{2}$. However, the wavelength for reentry (conduction velocity $\mathrm{x}$ action potential duration) was different between the two surfaces (Table 1), $6.6 \mathrm{~cm}$ on the endocardium versus $5.3 \mathrm{~cm}$ on the epicardium. This was due to action potential duration being shorter in the epicardium than in the endocardium while minimum conduction velocity remained constant.

Table 1. Action potential duration at $90 \%$ repolarization (APD), minimum conduction velocity (CV), and wavelength for reentry in the LV tissue model.

\begin{tabular}{lcc}
\hline \hline Parameters & Endocardium & Epicardium \\
\hline & 300 & 240 \\
APD (ms) & 22 & 22 \\
Minimum CV (cm/s) & 6.6 & 5.3 \\
Wavelength $(\mathrm{cm})$ & & \\
\end{tabular}

When stimulating the entire epicardial, endocardial, or both surfaces with the defibrillation protocol, the minimum stimulus required to terminate VF differed (Top of Table 2). On the epicardial or endocardial surfaces only, it required $>512$-fold the capture threshold, or $>7,168$ 
$\mu \mathrm{A} / \mathrm{cm}^{2}$, which is becoming a strong shock. However, when stimulating both surfaces at the same time, only 16fold $\left(224 \mu \mathrm{A} / \mathrm{cm}^{2}\right)$ threshold capture was needed to defibrillate the LV model, which is a remarkable $97 \%$ decrease from single surface stimulation.

Table 2. Stimulus threshold factors for different surfaces and transmural depths to defibrillate the LV tissue model.

\begin{tabular}{lcc}
\hline \hline Surface & Threshold factor & Current $\left(\mu \mathrm{A} / \mathrm{cm}^{2}\right)$ \\
\hline Surface only & & \\
Endocardium & $>512$ & $>7168$ \\
Epicardium & $>512$ & $>7168$ \\
Both & 16 & 224 \\
& & \\
$50 \%$ depth & & 112 \\
Endocardium & 8 & 224 \\
Epicardium & 16 & 14 \\
Both & 1 & \\
\hline \hline
\end{tabular}

Figure 2.A illustrates the failure of epicardial surface stimulation at 16-fold capture threshold to defibrillate the LV model. The epicardial surface stimuli slows down the scroll wave in the epicardium, but not the endocardium, for which over time the two synchronize again with one another to continue sustained reentrant activity. Figure 2.B shows how when stimulating both the epicardial and endocardial surfaces at the same time with 16-fold capture threshold, the scroll wave underlying the VF is rapidly terminated.

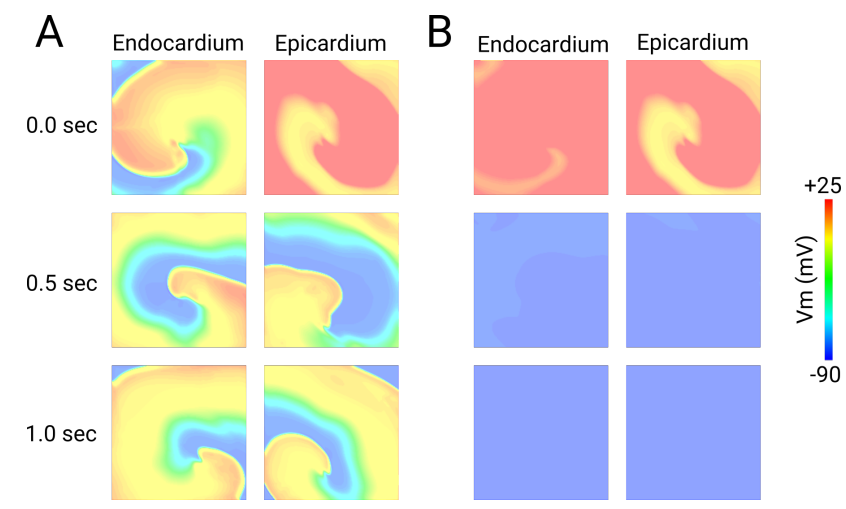

Figure 2. Defibrillation in LV wedge model with stimulus 16-fold capture threshold. A. S3 stimulus applied to the epicardial surface only which failed to terminate VF. $B$. S3 stimulus applied to both the endocardial and epicardial surfaces which successfully terminated VF.

Lastly, when stimulating halfway to the mid-myocardium from the endocardial and epicardial surfaces, the stimulus current needed to defibrillate the LV tissue model was dras- tically reduced (Bottom of Table 2). Note, the threshold factor for defibrillation with endocardial stimulation was half that for epicardial stimulation ( 8 vs 16 ), which corresponds with the longer wavelength for reentry in the endocardium versus epicardium.

\section{Conclusions}

Ventricular surface stimulation is feasible for terminating VF. However, it is dependent on the stimulus strength, depth, and targeted surface. According to this simulation study, it is best to target both the endocardial and epicardium surfaces, which allows to use a much smaller stimulus strength for robust defibrillation $(97 \%$ less current) than on a single surface. Stimuli penetrating deeper into the LV wall significantly reduces stimulus strength necessary for defibrillation. For these deeper surface stimuli, the endocardium requires a slightly less stimulus current to terminate VF due to having a longer wavelength for reentry.

Although useful, these findings were obtained in a simplified monodomain model. Future studies will investigate the effects of virtual electrode depolarization and electrode shape/size in a more computationally expensive bidomain model, as well as, determine if the presence of Purkinje fibers lowers the threshold for defibrillation the endocardium. A wide-array of arrhythmias, such as polymorphic and monomorphic tachycardia, and VF from single and multiple stationary and drifting reentrant sources will also be investigated.

Lastly, optical mapping experiments in intact swine hearts are currently being performed to validate the simulation results and the effects of surface stimulation on VF models. To help guide future VF electrotherapies, optical imaging of this study repeated in swine LV wedge preparations should help determine if these stimulus strengths are feasible in living cardiac tissue, as well as if they are below both the pain and damage thresholds for electrical shocks.

\section{Acknowledgments}

This research was funded by the French National Research Agency grants ANR-10-IAHU-04 and ANR-16CE19-0009. C. Hawks was supported by the Asociación de Amigos de UNAV, Obra Social "la Caixa" y Fundación Bancaria Caja Navarra, and Proyecto SAF2014-58286-C22-R. Simulations were performed on the HPC platform AVAKAS maintained by the Mésocentre de Calcul Intensif Aquitain (MCIA) of the Université de Bordeaux, France

\section{References}

[1] Priori S.G., et al. Task force on sudden cardiac death of the european society of cardiology. Eur Heart J 2001;22:1274450. 
[2] Doubert J.P., et al. Inappropriate implantable cardioverterdefibrillator shocks in MADIT II: frequency, mechanisms, predictors, and survival impact. J Am Coll Cardiol 2008;51:1357-65.

[3] Karanthanos T.V., et al. Opsin spectral sensitivity determines the effectiveness of optogenetic termination of ventricular fibrillation in the human heart: a simulation study. J Physiol 2016;594:6879-6891.

[4] Gutbrod S.R, et al. Patient-Specific flexible and stretchable devices for cardiac diagnostics and therapy. Prog Biophys Mol Biol 2014;115:244-251.

[5] Trayanova N.A., et al. Computational cardiology: how computer simulations could be used to develop new therapies and advance existing ones. Europace 2012;14:v82v89.

[6] Bayer J.D., et al. A novel rule-based algorithm for assigning myocardial fiber orientation to computational heart models. Ann Biomed Eng 2012;40:2243-54.

[7] Bayer J.D. et al. Mechanisms linking electrical alternans and clinical ventricular arrhythmia in human heart failure. Heart Rhythm 2016;13:1922-32.
[8] ten Tusscher K.H.W.J., et al. Alternans and spiral breakup in a human ventricular tissue model. Am J Physiol Heart Circ Physiol 2006;291:H1088-H1100.

[9] Gima K, et al. Ionic current basis of electrocardiographic waveforms: a model study. Circulation 2002;90:889-896.

[10] Berenfeld O. et al. Spatially distributed dominant excitation frequencies reveal hidden organization in atrial fibrillation in the Langendorff-perfused sheep heart. J Cardiovasc Electrophysiol 2000;11:869-79.

[11] Stewart A.J. et al. Frequency analysis of ventricular fibrillation and resuscitation success. $Q \mathrm{~J}$ Med 1992;85:761-9.

Address for correspondence:

Jason D. Bayer

IHU LIRYC - Campus Xavier Arnozan

Avenue du Haut Leveque

33600 Pessac

France

jason.bayer@ihu-liryc.fr 